\title{
Pratiques
}

Linguistique, littérature, didactique

\section{Les albums documentaires sur les peintres : un exemple d'interdisciplinarité au cycle 3 de l'école primaire}

Documentary albums on painters : an interdisciplinary example in cycle 3 of french primary school

\section{Anne Leclaire-Halté et Luc Maisonneuve}

\section{OpenEdition}

\section{Journals}

Édition électronique

URL : http://journals.openedition.org/pratiques/3611

DOI : $10.4000 /$ pratiques.3611

ISSN : 2425-2042

Éditeur

Centre de recherche sur les médiations (CREM)

\section{Référence électronique}

Anne Leclaire-Halté et Luc Maisonneuve, «Les albums documentaires sur les peintres : un exemple d'interdisciplinarité au cycle 3 de l'école primaire », Pratiques [En ligne], 175-176 | 2017, mis en ligne le 22 décembre 2017, consulté le 01 mai 2019. URL : http://journals.openedition.org/pratiques/3611 ; DOI : 10.4000/pratiques.3611

Ce document a été généré automatiquement le 1 mai 2019.

(c) Tous droits réservés 


\title{
Les albums documentaires sur les peintres : un exemple d'interdisciplinarité au cycle 3 de l'école primaire
}

\author{
Documentary albums on painters : an interdisciplinary example in cycle 3 of \\ french primary school
}

Anne Leclaire-Halté et Luc Maisonneuve

1 Les nouveaux programmes de 2015 incitent les enseignants de l'école primaire à l'interdisciplinarité, et, dans le domaine qui est celui de ce numéro de Pratiques, aux relations entre français (plus particulièrement lecture/écriture) et arts plastiques. En effet, dans la rubrique «Culture littéraire et artistique» du programme du cycle 3, on trouve la notation suivante, qui invite à des variations sémiotiques quant aux supports de lecture proposés aux élèves : « en CM1 et CM2, on veille à varier les genres, les formes et les modes d'expression (texte seul, texte et image pour les albums et la bande dessinée, image animée pour les films)»(2015, p. 121). Dans la rubrique "Croisement des enseignements ", les enseignants sont invités également à faire réfléchir leurs élèves au traitement de ces supports: "outre la recherche d'informations, le traitement et l'appropriation de ces informations font l'objet d'un apprentissage spécifique, en lien avec le développement des compétences de lecture et d'écriture » (ibid., p. 126). En arts plastiques enfin, une compétence à travailler est la sensibilité aux questions artistiques, la description des œuvres d'art faisant partie de cette compétence. Des mises en relation interdisciplinaires entre français et arts plastiques sont donc attendues.

2 Ces nouveaux programmes, associés au fait que les auteurs de cet article s'intéressent à la relation texte-image dans les ouvrages pour la jeunesse (Leclaire-Halté \& Maisonneuve, 2016) et aux problèmes de lecture que peuvent rencontrer les élèves dans la lecture des documents composites ${ }^{1}$, expliquent l'objet de ces lignes. Il y sera question, en effet, de la façon dont des albums documentaires sur les peintres peuvent être introduits en classe, 
et, plus spécifiquement, de la manière dont une double page (puisque c'est l'unité de sens la plus communément présente dans ce genre d'ouvrage) de ces albums est conçue et lue. À partir d'un exemple, nous étudierons dans quelle mesure une activité de lectureécriture peut à la fois favoriser la compréhension en lecture des doubles pages d'albums qui nous semblent relever d'un genre particulier, les albums documentaires sur les peintres, et sensibiliser les élèves à la description d'œuvres d'art.

Qu'est-ce qu'un album documentaire consacré à un peintre? Nous désignons ainsi ces livres spécifiquement destinés aux jeunes lecteurs, que l'on trouve dans les librairies de musées ou dans les rayons de librairies spécialisés en littérature de jeunesse, qui ont pour vocation de faire connaitre un peintre et son œuvre. Ces ouvrages sont une forme que peut prendre la médiation culturelle (Caillet et al., 2016), il s'agit alors d'une médiation indirecte (Bordeaux, 2008), et il est du ressort de l'école d'en favoriser la lecture par les élèves. Plus spécifiquement, ce sont des textes illustrés².

4 Les relations entre littérature de jeunesse et arts, la première étant conçue comme une médiation possible vers les seconds, sont en plein développement, si l'on en croit la quantité de collections offrant des ouvrages écrits pour la jeunesse relevant de ce genre. Cette production abondante a d'ailleurs fait l'objet de dossiers dans deux numéros de $\mathrm{La}$ Revue des Livres pour enfants: le numéro 246 paru en 2009, et le numéro 272, plus récent, paru en septembre 20133. Dans le numéro 246, un court article de S. Curtil (2009) montre le flou qui existe entre différentes appellations: livre d'art, livres d'artistes, livres d'artistes pour enfants. Pour notre part, nous n'entrerons pas dans ces distinctions, qui posent effectivement de nombreuses questions, mais nous nous centrerons sur ces ouvrages documentaires tout entiers consacrés à un peintre et se donnant pour objectif de sensibiliser les jeunes lecteurs à la production de ce dernier, par un choix de reproductions de ses œuvres. Ces ouvrages ont pour objectifs d'apporter des informations sur un peintre et son œuvre, de plaire, mais aussi de créer un habitus culturel.

5 Parmi tous les albums documentaires sur les peintres, ceux consacrés à H. Matisse ont été choisis : c'est, avec P. Picasso, l'artiste du XXe siècle dont l'œuvre est sans doute la plus divulguée par de nombreux documentaires pour la jeunesse. Et, dans la production abondante de cet artiste, il sera question des doubles pages consacrées à un tableau, $L a$ danse, qu'on trouve présent dans de nombreux documentaires sur ce peintre. C'est que ce tableau est doublement emblématique au sens où l'entend S. Tisseron (1998, p. 143) : une image emblématique est une image dont on a "[enlevé] les détails", une image décontextualisée, «soit par le choix du cadrage, soit par le travail sur l'image». Pour $\mathrm{S}$. Tisseron, l'emblème tend par conséquent vers une certaine forme d'abstraction. Et $\mathrm{La}$ danse est bien une abstraction : les danseuses représentées ne sont pas réalistes, ce sont des « épures» de danseuses ${ }^{4}$. S. Tisseron (1998, p. 131) ajoute par ailleurs que «l'une des conditions pour qu'une image devienne emblématique (ça n'est pas la condition absolue), c'est évidemment qu'un groupe la reconnaisse comme emblématique pour lui ». Ce tableau devient donc emblématique du travail d'H. Matisse de par la forme abstraite de sa réalisation et parce qu'il est reconnu comme tel par la communauté des amateurs et critiques d'H. Matisse.

6 Après une approche rapide de la présentation du tableau La danse dans trois doubles pages extraites de trois albums documentaires de jeunesse consacrés à H. Matisse, nous comparerons ces présentations à celles réalisées par une classe de $\mathrm{CM} 2^{5}$, dans le cadre d'une séquence qui sera décrite plus loin. À l'issue de cette étude, nous nous interrogerons sur les intérêts et les limites d'un tel travail interdisciplinaire. 


\section{Descriptif des trois doubles pages} dernier. Dans MAT il n'y a pas de titre pour le tableau : cette absence peut s'expliquer par le fait que l'ouvrage s'adresse, en priorité, à de très jeunes lecteurs. Dans MSP, il est par contre très précis et se rapproche du cartel du musée, indiquant le matériau, la taille, la date, le lieu d'exposition habituel : «Henri Matisse, La danse, 1909-1910. Huile sur toile, 260x391cm. St Pétersbourg, musée de l'Ermitage ». Dans MPM, il est moins complet, « La danse 1909, St Pétersbourg, musée de l'Ermitage ». Quoi qu'il en soit, la présence de ces informations peut instaurer de la connivence avec le lecteur cultivé et/ou être considérée aussi comme une initiation à la culture muséale.

Qu'en est-il du titre du texte qui accompagne le tableau ? Dans MAT, il s'agit d'une phrase en couleur contrastant avec le noir des caractères du texte : «Pour célébrer le bonheur de vivre, rien de mieux qu'une farandole». Dans MPM, le titre se fait métaphorique, «Les corps décollent ». Enfin, dans MSP, « Le rythme dans le pot » est le titre général pour les œuvres de la double page consacrée à la danse ; «Ballet russe » est le titre du texte se rapportant spécifiquement au tableau La danse. Les deux titres reposent sur un jeu de 
mots, comme d'ailleurs le titre de l'album documentaire lui-même ${ }^{7}$. Ainsi se manifeste, aux yeux du jeune lecteur, le fait que l'art n'est pas synonyme de sérieux, et que l'on peut s'amuser en parlant de peinture.

\section{Le rapport texte-image}

13 Pour F. Rigat (2005, p. 154), le texte expographique, celui qui s'affiche dans les musées, a une double fonction: «non seulement il décrit l'objet ou l'ensemble des objets exposés, mais surtout il montre que celui-ci est doté de nombreuses qualités, qu'il vaut le déplacement. Outre cette fonction spécifique, les textes expographiques ont un autre rôle, tout aussi essentiel : aider le visiteur à comprendre ce qui lui est montré ».

On peut faire l'hypothèse que, même si le contexte n'est pas le même et que le visiteur est en fait un lecteur, le texte des doubles pages étudiées remplit la triple fonction attribuée aux textes expographiques: décrire La danse, aider le lecteur à comprendre ce tableau, enfin le valoriser. Ces textes présentent en effet une triple dimension: descriptive, interprétative (de l'ordre du commentaire), et enfin émotionnelle, plus particulièrement laudative.

\section{Que décrire du tableau?}

Cinq femmes se donnent la main pour une ronde joyeuse.

On les voit bondir et rebondir, pleines d'énergie.

Les corps, immenses et orangés, se découpent sur le ciel bleu et la colline verte.

Trois couleurs qui résonnent gaiement entre elles, comme trois notes de musique !

\section{MSP : Ballet russe}

En 1909, le collectionneur russe Chtchoukine commande à Matisse «la danse » et «la Musique ». Dans la première il reprend la farandole qu'il avait peinte dans « le Bonheur de vivre » quelques années plus tôt. L'œuvre n'est réalisée qu'avec trois couleurs mais quelles couleurs! Riches, saturées, méditerranéennes, elles donnent le tempo...

Cependant pas facile de différencier les personnages. Savez-vous s'il s'agit de baigneuses, de danseuses ou de nymphes? Non! Logique puisque les personnages sont surtout là pour incarner toutes sortes de mouvements : se cambrer, se replier, sautiller, s'élancer, glisser. Ça bouge dans cette toile et ça donne plutôt envie de rentrer dans la ronde. Les artistes russes du $\mathrm{XX}^{\mathrm{e}}$ siècle suivront le rythme de ce tableau comme Malevitch et son baigneur.

MPM : Les corps décollent

Rouge des corps, vert de l'herbe, bleu du ciel.

Une ronde primitive se déploie sur un grand panneau de 4 mètres de long.

Les corps des danseurs sont lisses et puissants.

Les pieds martèlent le sol, les bras se tendent, les bustes se cambrent, se penchent, s'étirent.

Ça tourne, ça va vite, de plus en plus vite.

Les créatures sont possédées par l'esprit de la danse. Leurs pieds quittent le sol, elles s'envolent, elles décollent. Elles sont dans la danse.

C'est un riche collectionneur russe qui a commandé ce grand panneau décoratif à Matisse pour orner l'escalier de son bel hôtel particulier de Moscou. Il ne s'attendait pas à une 
telle composition. En, la découvrant, il se demande ce qu'il doit en penser. Et puis il finit par s'y faire et même à beaucoup l'aimer.

L'art c'est souvent comme ça, il faut se donner le temps de l'apprivoiser.

Dans ces documentaires, l'image est première et le texte, qui occupe moins de place que l'image, se trouve dans un rapport de subordination par rapport à celle-ci. Il assure la médiation entre le tableau reproduit et le jeune lecteur. Il peut se comprendre sans le tableau, mais il est dans un rapport de description par rapport à ce dernier. Un certain nombre de déictiques renvoient d'ailleurs à La danse, indiquant que l'énonciateur a le tableau sous les yeux et qu'il suppose qu'il en est de même pour le lecteur. Il s'agit de déterminants définis (MAT: «Les corps, immenses et orangés, se découpent sur le ciel bleu et la colline verte »; MSP : « les personnages ») ou de démonstratifs (MSP : « ça bouge dans cette toile »).

Que disent ces textes au jeune lecteur? P. Galluzzi (2000, p. 134) énumère un certain nombre d'éléments éventuellement présents dans un texte associé à un tableau :

Il est possible de présenter chaque œuvre à la lumière des connaissances factuelles et intellectuelles qui en éclairent le sens, de la replacer dans la biographie de son auteur, d'en expliquer sa technique d'exécution, la composition, l'histoire, les motivations du commanditaire, de la rapprocher des documents liés à sa genèse et à sa fortune, des dessins préparatoires, des modèles d'où est tirée son inspiration, des imitations qui en ont été faites, etc.

Les notions d'histoire des arts, convoquées pour décrire et commenter les tableaux à des adultes (voir par exemple P. Schneider ${ }^{8}, 1992$ ), ne sont pas à l'ordre du jour dans ces ouvrages qui s'adressent aux enfants. Cependant, les textes délivrent des informations. La grille d'analyse suivante, construite à partir de la citation de P. Galluzzi, synthétise les éléments contextuels (éléments biographiques ou historiques), les éléments descriptifs et formels (description générale, éléments techniques, allusions au processus de création), les éléments d'inscription dans le temps (mention d'un avant et/ou d'un après le tableau).

Tableau 1. Ce que les textes disent de La danse

\begin{tabular}{|c|c|c|c|}
\hline & $\begin{array}{l}\text { Éléments } \\
\text { contextuels } \\
\text { informatifs }\end{array}$ & Éléments descriptifs et formels & $\begin{array}{l}\text { Inscription dans le } \\
\text { temps }\end{array}$ \\
\hline MAT & & $\begin{array}{l}\text { «farandole », « ronde joyeuse » } \\
\text { « } 5 \text { femmes » } \\
\text { « orangés », « bleu », « verte " } \\
\text { «bondir » « rebondir » }\end{array}$ & \\
\hline MSP & $\begin{array}{l}1909 \\
\text { collectionneur russe } \\
\text { Chtchoukine }\end{array}$ & 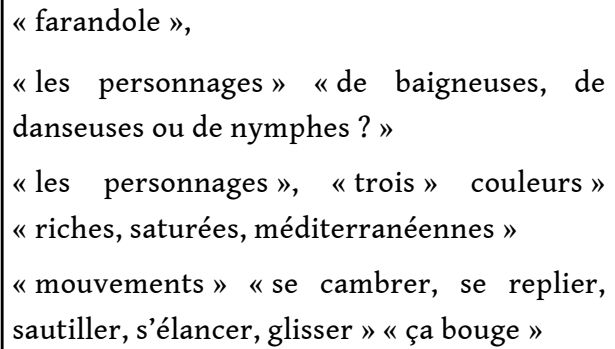 & $\begin{array}{l}\text { Avant: «le } \\
\text { bonheur de vivre " } \\
\text { Après : «Malévitch } \\
\text { et son baigneur » }\end{array}$ \\
\hline
\end{tabular}




\begin{tabular}{|c|c|c|}
\hline MPM & $\begin{array}{l}\text { Riche collectionneur } \\
\text { russe } \\
\text { Moscou }\end{array}$ & 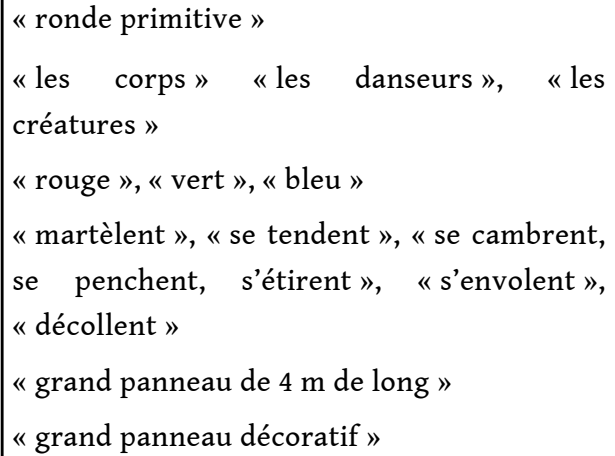 \\
\hline
\end{tabular}

Deux textes sur trois présentent un élément contextuel, le même biographème: H. Matisse a travaillé pour S. Chtchoukine. MSP est plus précis que MPM sur ce point: l'année de la commande et le nom du commanditaire sont donnés. Mais les trois textes sont relativement avares d'informations sur le peintre, soit qu'elles ont été données ailleurs (les doubles pages sont des extraits d'ouvrages complets), soit que leur rédacteur estime qu'il est inutile de fournir au jeune lecteur plus d'éléments biographiques qu'indiqué. Les mêmes éléments descriptifs apparaissent dans tous les documentaires, la danse, les personnages, les couleurs, le mouvement, avec toutefois des différences d'un texte à l'autre. Par exemple, dans MPM il est question de "ronde primitive ", là où sont mentionnées, dans les autres textes, une farandole et une ronde. De même, les personnages ne sont pas présentés de la même manière. Dans MPM ils sont évoqués de manière asexuée, « les danseurs ", alors qu'il est question de "femmes » dans MAT et que le terme plus technique et plus neutre de " personnages » est utilisé dans MSP. Les limites de cet article ne nous permettent pas une analyse linguistique détaillée du choix de ces éléments descriptifs ; mais on peut noter qu'ils traduisent la subjectivité du regard de l'auteur de la description.

Enfin, seul MSP situe l'œuvre dans le temps, avec un «avant » et un «après ». Mais le renvoi à "Malévitch et son baigneur", pour signifier l'influence d'H. Matisse sur la peinture ultérieure, ne peut guère avoir de sens pour les jeunes lecteurs sans l'accompagnement d'un médiateur.

\section{Dimension interprétative}

La frontière entre descriptif et interprétatif est souvent difficile à définir. C'est particulièrement net dans la façon dont les couleurs sont traitées dans les textes. Dans deux ouvrages, elles sont attribuées «naturellement » à des éléments du paysage : le bleu est celui du ciel (MAT, MPM), le vert est celui d'une colline (MAT), de l'herbe (MPM). Seul MSP ne se prononce pas à ce sujet. À propos des personnages, si MAT et MSP considèrent que ce sont des femmes, MPM parle de "danseurs»; et, dans MSP, l'identité des personnages de la ronde fait l'objet d'un commentaire sous forme de question : "Savezvous s'il s'agit de baigneuses, de danseuses ou de nymphes?». Dans MPM, ces danseuses sont celles d'une danse "primitive », ces "créatures sont possédées par l'esprit de la danse " : le tableau est lu comme une fête rituelle renvoyant à une humanité primitive se livrant à des rites de possession. Dans les trois textes, si l'on met de côté ces différences, il existe cependant un point commun : l'accent mis sur la joie, l'énergie, le mouvement que dégage le tableau. P. Schneider (1992), quant à lui, met l'accent sur l'opposition, au sein 
de cette danse mi-ronde mi-farandole, entre deux ordres : l'ordre humain des conflits et des déséquilibres, et un ordre supérieur qui serait un ordre idéal, si les personnages avaient formé une figure parfaite. «Ainsi, La danse nous montre simultanément deux ordres différents: la figure parfaite, géométrique (l'ovale) perçue à travers l'activité tumultueuse des figurants » (ibid., p. 285). Cette opposition n'est reprise par aucun des trois textes des documentaires, qui, cependant, insistent bien sur l'impression de mouvement et d'énergie dans le tableau.

\section{Une lecture subjective du tableau}

La technique picturale est peu présente dans ces textes, au profit de la subjectivité des auteurs, de l'émotion procurée par le tableau. Certes, un vocabulaire spécialisé est parfois utilisé dans deux textes sur trois: dans MSP, on trouve "toile ", "tableau ", " couleurs saturées", alors que dans MPM il est question de "panneau décoratif», de " composition ». Mais les textes valorisent surtout l'énergie et la joie du tableau grâce à des adjectifs et des adverbes axiologiquement positifs, des phrases exclamatives, des énumérations de verbes d'action: "trois couleurs qui résonnent gaiement entre elles, comme trois notes de musique !» (MAT), « les pieds martèlent le sol, les bras se tendent, les bustes se cambrent, se penchent, s'étirent » (MPM). Et, pour entrainer le jeune lecteur dans l'admiration du tableau, les textes se veulent proches de lui. MSP n'hésite pas à s'adresser directement aux lecteurs: «savez-vous que...? ». Fuir l'ennui que pourrait procurer la lecture de ce genre de textes passe aussi par l'emploi d'énoncés écrits de style parlé : «ça bouge dans cette toile » (MSP), «l'art, c'est souvent comme ça, il faut se donner le temps de l'apprivoiser" (MPM). Cette dernière formule, qui clôt le texte, généralise l'expérience de $\mathrm{S}$.Chtchoukine, décontenancé d'abord par le tableau d'H. Matisse d'après l'auteur de MPM. Adressée au jeune lecteur, elle vise à inclure ce dernier dans la communauté des amateurs d'art.

Ainsi, une image du regard sur l'art, subjectif plutôt que savant, est donnée par ces textes, qui insistent sur les émotions ressenties à la vue du tableau plus que sur une approche analytique, technique. En cela, ils sont en accord avec «les pratiques culturelles de médiation [qui] mettent en avant les aspects émotionnels, les dimensions de la sensibilité, du corps, du geste, de la créativité, porteurs de signification existentielle et relationnelle » (Montandon \& Perez-Roux, 2014, p. 9-10).

\section{Des textes eux-mêmes une œuvre d'art?}

Les textes de ces albums font l'objet d'une mise en page très soignée. M. Sellier (2009, p. 130), l'auteure de MPM, note toute l'attention qu'elle porte au rapport texte-image :

Ce rapport texte-image que j'ai découvert lorsque j'étais journaliste à Bayard presse, cette complémentarité sans redondance, je l'ai travaillée, je la travaille toujours avec passion dans chacune de mes collections. Un livre d'art est un tout. L'écrit et le visuel doivent s'agencer harmonieusement. Ainsi je veille constamment au bon déroulé du texte, à la pertinence des césures, des passages à la ligne, si importants pour le rythme de lecture.

C'est l'image du texte dont il est ici question (Souchier, 1998), avec une prise en compte de la matérialité de celui-ci (papier support, typographie, mise en page). Dans MPM, le texte se déroule sur un fond gris (dans cet album, la couleur du fond change à chaque double page), le titre aligne des caractères en forme de vague, certains mots du texte sont 
mis en avant avec l'utilisation de caractères d'une taille plus grande. Il est, par ailleurs, et contrairement aux deux autres albums, situé sur la page de gauche, donc offert en premier au regard du lecteur. Dans MAT, sur un fond blanc, le titre reprend la couleur orangée des corps du tableau. MSP, enfin, utilise également des couleurs différentes pour les titres. Ce dernier album présente aussi deux dessins colorés qui viennent orner soit le titre de la double page, soit les références des tableaux.

Pour résumer les caractéristiques de ces doubles pages qui seront ensuite soumises à des élèves de cycle 3 , elles associent la reproduction de La danse et un texte tout à la fois descriptif, interprétatif et subjectif, cela en une mise en page soignée où la couleur a toute son importance, comme dans l'œuvre picturale d'H. Matisse. Les textes proposés sont en fait un mélange tout à la fois de descriptions, de commentaires, d'impressions, de sentiments, et en cela, ils illustrent la diversité que peuvent présenter les écrits sur l'art (Vaugeois, 2005) et les difficultés à les circonscrire génériquement. Dans deux cas sur trois, ils apportent des informations sur le peintre. Bien que succinctes, en liant l'œuvre à la vie de l'artiste, ces informations contribuent à ancrer celui-ci dans la vie quotidienne' Les textes fournissent également des renseignements sur le tableau tout en le louant, en attirant l'attention sur les couleurs, le mouvement, le rythme du tableau, en mettant en avant le talent de son auteur, MSP étant sans doute le plus enclin à ces éloges. Enfin, les trois albums, en insistant sur les émotions ressenties à la vue du tableau, d'une part visent à éduquer la sensibilité artistique des jeunes lecteurs pour les inclure dans la communauté des amateurs d'art, d'autre part, contraignent quelque peu les élèves dans la manifestation de leurs propres émotions. Ainsi, par exemple, au cours de cette séquence, aucun élève ne dira ne pas avoir aimé tout ou partie du tableau.

\section{La séquence réalisée en classe}

31 Les doubles pages analysées ci-dessus ont fait l'objet d'une séquence réalisée dans une classe de CM2 de 26 élèves $^{10}$, dans une école située dans une banlieue résidentielle de Rennes. L'enseignante de la classe, en proposant cette séance, se situe au croisement de deux disciplines. Elle a d'abord visé un objectif relevant de la didactique du français. En effet, les élèves avaient à réaliser, pour le site internet de l'école, une double page de présentation de $L a$ danse d'H. Matisse, à la manière de trois doubles pages d'albums étudiés en classe, doubles pages des albums précédemment analysés. La didactique du français pouvant être définie, au sens large, comme le fait de faciliter, pour tous les élèves, « la réception et la production des discours oraux et écrits » (Halté, 2008), cette séquence, visant à amener les élèves à lire et à comprendre les supports spécifiques que constituent les ouvrages documentaires sur les peintres, tout en articulant activités de lecture et d'écriture ${ }^{11}$, s'inscrit bien dans cette perspective. L'enseignante visait aussi, en un objectif relevant cette fois de l'histoire des arts, l'acquisition par les élèves de connaissances sur $\mathrm{H}$. Matisse et sur le tableau La danse : ils avaient à décrire ce tableau et à porter un regard critique sur lui, à découvrir H. Matisse, à acquérir quelques termes spécifiques à l'univers de la peinture.

La séquence a comporté six séances. Après un tri d'ouvrages (albums de jeunesse) devant permettre de caractériser les ouvrages documentaires de jeunesse sur un peintre (séance 1), La danse a été projeté aux élèves, qui, individuellement, en ont rédigé une description écrite puis ont lu à la classe leur production (séance 2). La séance 3 a consisté en une présentation des trois doubles pages des albums documentaires; les élèves ont ensuite 
indiqué laquelle ils préféraient. Une quatrième séance a permis une comparaison plus poussée des trois doubles pages (la mise en page, le texte, les titres). Ce travail a été suivi, en deux séances ultérieures, d'un retour sur les textes produits initialement et l'élaboration, cette fois en binôme, pour le site internet de l'école, d'une double page présentant le tableau et intégrant des textes initiaux éventuellement modifiés.

Nous ne commenterons que trois des principaux épisodes de cette séquence, en articulant les deux derniers: les préférences des élèves et l'écriture des premiers textes, ceci en relation avec la réalisation des doubles pages qui constitue le troisième épisode. Ces trois épisodes nous semblent en effet particulièrement pertinents. Le premier met en avant ce qui parait le plus important et le plus intéressant pour les élèves dans les albums retenus pour cette séquence. La mise en relation du deuxième et du troisième épisode permet d'une part d'analyser ce qui est modifié lors de la réalisation des doubles pages par rapport aux textes initiaux, et d'autre part de mesurer, en partie, ce qui a été retenu de la comparaison de ces doubles pages consacrées au tableau de Matisse.

\section{Les préférences des élèves}

Seize élèves préfèrent MSP, sept MPM, trois MAT. Ils justifient ces choix de la manière suivante :

Tableau 2. Justification des préférences des élèves

\begin{tabular}{|c|c|c|c|}
\hline & MSP (16 él.) & MPM (7 él.) & MAT (3 él.) \\
\hline Titre & Jeu avec les mots du titre & Titre en zigzag (2 él.) & Gros titres \\
\hline Texte & $\begin{array}{l}\text { Beaucoup de texte ( } 2 \text { él.) } \\
\text { Éléments qu'on ne visualise pas («le } \\
\text { texte parle de... »): il donne des } \\
\text { indications sur les nymphes, } \\
\text { l'acheteur ( } 7 \text { él.) }\end{array}$ & $\begin{array}{l}\text { Précisions sur le } \\
\text { collectionneur (3 él.) } \\
\text { Texte donne envie de lire }\end{array}$ & $\begin{array}{l}\text { Léger } \\
\text { beaucoup de } \\
\text { texte) } \\
\text { Écrit en gros } \\
\text { Un petit poème } \\
\text { en rouge } \\
\text { Explique/décrit } \\
\text { bien }\end{array}$ \\
\hline $\begin{array}{l}\text { Mise en } \\
\text { page }\end{array}$ & 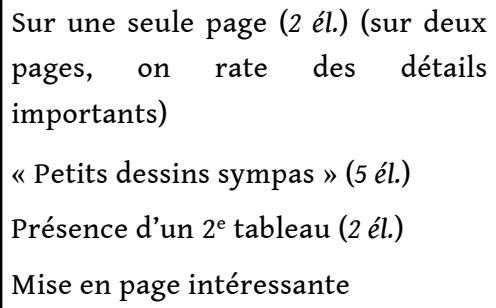 & $\begin{array}{l}\text { Tableau mis en avant (au } 1^{\mathrm{er}} \\
\text { plan) (4 él.) (plus grande } \\
\text { surface du tableau) } \\
\text { Mise en page (2 él.) } \\
\text { Moins de choses }\end{array}$ & $\begin{array}{l}\text { Mise en page } \\
\text { «Il manque un } \\
\text { peu de décoration } \\
\text { autour» }\end{array}$ \\
\hline Divers & $\begin{array}{l}\text { Plus de couleurs (8 él.) } \\
\text { Joyeux (4 él.) }\end{array}$ & $\begin{array}{l}\text { Police d'écriture variée (3 él. } \\
\text { ) }\end{array}$ & Couleurs \\
\hline
\end{tabular}

L'activité appelle une remarque préalable, liée au vocabulaire utilisé par les élèves. Ce dernier traduit un certain usage d'une terminologie en rapport avec les albums 
documentaires présentés : texte, mise en page, titre, tableau, collectionneur, peintre, premier plan, police, couleur. Si ces termes ne sont pas utilisés de manière précise, on peut toutefois souligner qu'ils ne le sont jamais de manière fautive. Les élèves savent donc décrire les objets observés.

Sur le fond, la lecture du tableau ci-dessus permet de remarquer que c'est l'album le plus sophistiqué (mise en page générale, et, pour le texte, choix énonciatifs, quantité d'écrit) qui est massivement préféré. MSP est un ouvrage écrit pour des lecteurs plus âgés que ceux de MAT (on peut rappeler que ce dernier vise les lecteurs de plus de 5 ans). Même si, aux dires de l'enseignante, le texte de MSP pose quelques problèmes de compréhension, il s'avère que les élèves de CM2 se sentent plus interpellés par cette double page que par les autres.

Enfin, l'observation de ces justifications montre que ces élèves font peu de liens explicites entre leurs remarques et le tableau d'H. Matisse. Ils disent par exemple apprécier le grand nombre de couleurs utilisées dans les albums sans que celles-ci soient mises en rapport avec celles du tableau. Il en va de même pour toutes les remarques portant sur la forme du texte (taille et choix de police, mise en page).

\section{Comparaison des productions initiales des élèves avec leurs productions finales}

Toutes les séances ont été filmées, et nous disposons de plusieurs des travaux écrits par les élèves, dont deux séries de productions sur lesquelles nous nous appuyons dans cet article : les vingt-six textes initiaux des élèves et les doubles pages pour le site internet, moins nombreuses car effectuées en binômes. Ces dernières interviennent en fin de séquence, après un travail sur les trois doubles pages documentaires dont il a été question dans la première partie de cet article. À noter cependant qu'entre les deux situations d'écriture, il n'y a pas eu d'étude précise du contenu des textes des albums, mais de la mise en page générale, des couleurs et des polices utilisées, etc. L'enseignante note d'ailleurs, a posteriori, qu'il aurait été bon de revenir sur les textes eux-mêmes, qui ont posé des problèmes de compréhension à certains élèves. Elle regrette de ne pas avoir pris le temps de traiter ce qu'elle relève comme les trois dimensions de ces textes, descriptive, informative, et interprétative ${ }^{12}$. Néanmoins, même si la consigne d'écriture n'est pas tout à fait la même (dans le premier cas « Décrivez le tableau à quelqu'un qui ne l'a jamais vu » et dans le second « Rédigez une double page pour le site internet de l'école »), même si les élèves ne sont pas placés dans la même situation par les injonctions de l'enseignante, nous revenons sur leurs premières productions pour voir dans quelle mesure, malgré l'absence de travail sur les textes des albums documentaires, ceux-ci ont influencé ces productions initiales pour la réalisation des pages pour le site internet.

\section{Analyse des textes initiaux}

Le premier jet, individuel, est produit dans les conditions suivantes : le tableau est projeté aux élèves, avertis du fait que leur texte sera utilisé pour une page internet présentant $L a$ danse sur le blog de l'école. Initialement, rien ne leur est indiqué sur la possibilité de donner leur avis ou de livrer leur sentiment sur La danse, mais une réponse positive sera donnée par l'enseignante en cours de tâche à une ou deux questions d'élève à ce sujet. 

mention de couleurs, la désignation des personnages (sexe, couleur, autre information prélevée), les lieux (associés à une couleur ou non), l'évocation du mouvement (présence ou non d'un terme générique pour désigner la danse, autre information sur le mouvement), l'interprétation (absence d'interprétation ou plutôt tendance à l'objectivité, interprétation donnée comme allant de soi, sans marquage énonciatif, interprétation donnée comme telle, avec un marquage énonciatif).

Presque tous les textes ont un titre, «La danse », ou " La danse de Matisse », suivi, souvent d'une phrase d'introduction avec le nom du tableau, l'auteur et la date, renseignements donnés initialement par l'enseignante. Tous les textes mentionnent deux couleurs, le bleu et le vert. Beaucoup en mentionnent plus, cependant seuls cinq textes accordent une importance particulière à la couleur. En ce qui concerne la désignation des personnages du tableau, la moitié des élèves de la classe parlent de cinq personnes ou personnages asexués. Un quart des élèves reconnait dans ces cinq personnages un certain nombre d'hommes et de femmes sans justifier ce qui leur permet de différencier leur sexe. Enfin cinq élèves identifient cinq femmes, un seul cinq hommes. Dans tous les cas, la nudité est presque toujours soulignée, sans autre commentaire. La couleur de la peau est aussi souvent relevée, orange, beige ; un élève la rapproche du bronze et un autre de l'argile. Quand un détail descriptif supplémentaire est donné, il s'agit, le plus souvent, de la couleur de la chevelure (marron, châtain). Enfin, deux élèves trouvent que ces personnages ont un gros ventre.

esonnages sont physiquement peu décrits, ils le sont davantage par leurs positions et mouvements. Mais le titre du tableau ayant été donné par l'enseignante avant l'observation, il était difficile de ne pas voir les personnages danser. Ainsi, près de la moitié des élèves note qu'ils se tiennent par la main, qu'ils forment une ronde, qu'ils dansent. Deux d'entre d'eux écrivent que les personnages volent. Plusieurs élèves vont un peu plus loin : trois perçoivent un cœur dans la forme de la ronde, deux mentionnent les mouvements de la danse, en ayant recours soit à la répétition du même verbe: «Ils tournent, tournent, tournent sans toucher l'eau " soit à l'énumération de verbes de mouvement différents : «Je les vois danser sur un sol vert, ils sautent, ils bougent ».

Enfin, deux élèves notent des éléments qui entrent en congruence avec l'analyse que fait P. Schneider (1992) du tableau. Ce dernier, en effet, décrit avec beaucoup de précision l'attitude de chaque danseur pour montrer la tension entre équilibre et déséquilibre de la ronde, tout comme le fait cet élève :

Sur le tableau on voit les personnes danser sur du vert. Chacun est placé différemment. Il y a cinq personnes dans la ronde. La première personne est de dos on aurait l'impression qu'elle va tomber. Puis à gauche d'elle il y a une personne qui va tourner. La troisième personne à gauche fait une pointe avec la jambe ; et met la jambe droite sur la gauche et regarde son ventre. La quatrième personne $a$ un pied en l'air. Et la dernière personne met un pied à l'intérieur du cercle. pieds ».

P. Schneider (1992, p. 284) relève, en d'autres termes, le même détail : «La danseuse à l'extrême gauche frappe si fortement du pied le sol qu'elle semble le fendre ».

46 Enfin, quelques-uns évoquent les émotions supposées ressenties par les personnages: "On dirait qu'ils ne veulent pas montrer leur tête»; "Les personnes n'ont pas l'air content d'être là ou encore elle a l'air de sourire ». 

écriture, ne correspond pas à la situation dans laquelle se trouve le lecteur d'une double page d'un album documentaire présentant un tableau d'H. Matisse. En effet, décrire un tableau pour quelqu'un qui ne l'a jamais vu, comme l'enseignante le demande initialement, et le décrire dans un ouvrage où le tableau figure en vis-à-vis du texte est différent. Mais décrire un tableau, qu'est-ce que cela signifie ? (Chabanne \& Dufays, 2011). En matière d'art, quelles sont les frontières entre description, commentaire, interprétation? Et quelle consigne d'écriture peut donner l'enseignante alors que l'étude des doubles pages n'a pas encore été effective? Il s'agissait de faire que les élèves s'expriment à propos d'un tableau, et le but est atteint.

Enseigner la parole sur l'œuvre, c'est justement se placer en deçà des formes savantes ou abouties que peuvent prendre les jeux de langage experts. C'est s'intéresser aux formes natives de cette parole, forcément hésitantes, balbutiantes : c'est être attentif à la genèse de l'expérience et de la parole esthétiques, genèse d'autant plus difficile qu'elle a affaire à du complexe et du labile. » (ibid., 2011, p. 16-17) " arbre ", " ile ", « continent »; pour le bleu, " ciel », " nuage », mais aussi " mer », « rivière », " eau ». Cela s'explique non seulement par les conventions associant objets et couleurs mais aussi par la position des personnages (qui marchent sur du vert et ont la tête dans le bleu). L'herbe et le ciel sont cependant les plus largement cités.

peuvent, très rarement, donner l'impression de ne se livrer à aucune interprétation: «Sur le tableau cinq personnes nues sont représentées en train de danser en ronde. En arrière-plan il y a du bleu et en dessous du vert où les personnages dansent dessus ».

Plus fréquemment, des éléments du tableau sont interprétés sans qu'il y ait marquage En arrière-plan on aperçoit le ciel bleu et la terre verte.

Presque tout le tableau est recouvert du ciel bleu.

Sur ce tableau, on y voit des personnes qui dansent, qui forment un cercle sur une ile.

On dirait qu'ils pourraient fermer les yeux.

vert représente la terre et le bleu la mer.

géante tout en bas.

On dirait qu'ils ne veulent pas montrer leurs têtes.

Les personnes n'ont pas l'air contentes d'être là.

fin de texte, détachée ou non par un « mon avis »: « je pense que », « je trouve que ». La première personne est lors souvent utilisée : «Je pense qu'ils sont sur un arbre parce que c'est rond et vert je le trouve très joli ».

Beaucoup plus rarement, un avis apparait au fil du texte, comme dans l'exemple suivant : «Cela est très beau car ils se tiennent tous la main ».

es textes des élèves laissent percevoir cette difficulté à dire la rencontre avec l'œuvre artistique, et en même temps, pour certains, manifestent une émotion ressentie à la vue 
du tableau. On s'interrogera tout de même sur l'absence de jugements dépréciatifs ou de notations de rejet, comme si l'école n'autorisait pas ce genre de remarques.

\section{Les doubles pages pour le site : que disent-elles de la lecture des doubles pages documentaires?}

Après l'étude comparative des doubles pages des albums, où l'attention des élèves s'est centrée sur la mise en page générale plus que sur les textes eux-mêmes (à propos de ces derniers, leurs questions ont traduit essentiellement des préoccupations lexicales à propos de termes comme "nymphe», " cambrer»), l'enseignante leur a demandé de réaliser, en binômes, leur propre double page pour le site internet de l'école. Elle a fourni à chaque groupe trois reproductions, de dimensions différentes, de La danse, une photo de S. Chtchoukine, une photo d'H. Matisse, la reproduction de La Joie de vivre, et divers documents élaborés par les élèves lors des séances antérieures, dont le texte initial. L'enseignante a rappelé aux élèves le destinataire de leur future réalisation et les observations faites lors de la comparaison des trois doubles pages dont ils devaient tenir compte: les places respectives du tableau et du texte, la présence ou non de dessins annexes sur la double page, les tailles des diverses polices utilisées, les couleurs, la présence de titres, etc.

Dans les treize doubles pages ainsi réalisées par les élèves, douze présentent un titre. Si quelques groupes restent fidèles à celui des textes du premier jet, «La danse » ou « La danse de Matisse ", d'autres optent pour une plus grande diversité, inspirée des titres dans les albums documentaires étudiés : «La danse à l'état pur », « Tous en rythme », « La vivacité de la danse », «La danse est dans la peau », « La joyeuse danse de Matisse », «La joie et le bonheur de danser », "Avec Matisse, ça danse!». L'importance du titre, pour rendre compte de la tonalité de l'œuvre, est ainsi marquée. Ces titres font souvent également l'objet d'un travail au niveau des couleurs et parfois de la typographie: par exemple, dans deux productions, ils forment un arc de cercle, préfigurant ainsi le thème du tableau.

Un autre élément remarquable est l'importance donnée par les élèves au caractère informatif de leur double page. En effet, contrairement aux documents étudiés, avares, d'un point de vue iconographique, en éléments purement informatifs, ils sont nombreux à y faire figurer les photos d'H. Matisse (dans onze cas) et de Tchouchkine (dans huit cas), accompagnées ou non d'une phrase biographique ou deux. Certes, cet apport d'informations a été induit par les documents fournis par l'enseignante, mais il traduit sans doute aussi une prise en compte par les élèves du lectorat du site, qui ne connait pas forcément $\mathrm{H}$. Matisse ni S.Chtchoukine. Deux groupes utilisent la reproduction du tableau antérieur La Joie de vivre, dont un groupe souligne l'importance en entourant d'un trait de couleur la farandole centrale, reprise dans La danse. Enfin, de nombreuses doubles pages comportent des dessins en couleur qui font partie de la mise en page effectuée par les élèves, éléments figuratifs (cœurs, crayon, cactus, coccinelle, fleurs etc.) ou nonfiguratifs (points et traits multicolores, petites spirales, points d'exclamation et d'interrogation...), en rapport ou non avec le tableau. MSP a été une source d'inspiration pour les élèves de ce point de vue.

Quant aux textes, ils ont fait l'objet de peu de modifications dans l'ensemble, par rapport à la première version. Cependant, de façon systématique, ils signalent tous que trois couleurs sont présentes: lors de la comparaison des trois doubles pages, un des rares 
points communs souligné a été en effet le fait que, dans les trois textes se rapportant au tableau, il était question de ces trois couleurs principales («L'expression vient de la surface colorée que le spectateur saisit dans son entier » a écrit H. Matisse [cité par Bauret, 1977, p. 31]). Trois textes parlent d'une "colline verte", comme dans MAT. Certains empruntent aux albums des mots ou des expressions : « Ils sont possédés par l'esprit de la danse », "Quatre mètres de long pour représenter le bonheur de vivre ", « leurs pieds commencent à décoller »...

Le sexe des personnages du tableau a suscité plusieurs interrogations de la part des élèves : si 6 textes sur 13 disent que ce sont des femmes, les autres ont recours au terme plus neutre de "personnes", ce qui semble signifier une prise de recul critique par rapport aux textes des documentaires, qui ne sont pas tous d'accord sur la question. Enfin, les textes de deux groupes, donnés ci-dessous, portent la trace de la lecture des textes des doubles pages, sur des points qui n'ont cependant pas été abordés en classe :

Avec Matisse ça danse.

En 1909 Matisse aime la danse! Toutes ces femmes nues font rêver le monde entier. Toutes, cheveux marron dans le vent, virevoltent en farandole et en musique. Court ou long peu importe. Seulement trois couleurs nous prennent aux yeux. La colline verte laisse entrevoir un grand fond bleu. Elles sont nues, grandes, joyeuses. Ce tableau est magnifique. Bravo Matisse !

Sur ce tableau nous pouvons apercevoir 5 personnes heureuses, qui dansent en formant une ronde. Les couleurs sont: bleu du ciel, vert de l'herbe et orange des corps. Les personnages font toutes sortes de figures.

Ça bouge, ça donne envie de danser. Nous avons l'impression que le tableau est fait pour être joyeux.

La manifestation de la subjectivité par la ponctuation exclamative, le recours au démonstratif «ça », l'insistance sur le caractère joyeux du tableau caractérisent en effet ces deux textes, alors que ni le rapport texte/tableau ni les caractéristiques linguistiques des textes n'ont été étudiés avec les élèves.

61 L'enjeu était d'entrer dans le concret d'un travail en classe avec une enseignante qui essaie de concilier des objectifs en histoire des arts et des objectifs de compréhension en lecture. L'analyse permet de faire un certain nombre de constats sur cette expérience interdisciplinaire, qui ne valent bien entendu que dans le contexte étudié, et de questionner l'intérêt et les limites de cette même expérience.

En matière de lecture, on peut supposer, à voir les productions finales, que les élèves se sont familiarisés avec les documentaires sur les peintres, sans doute plus, d'ailleurs, avec leur mise en page qu'avec les textes eux-mêmes, plus difficiles à caractériser. Ils ont aussi acquis des savoirs en histoire des arts, par exemple, l'existence d'H. Matisse, de La danse, $\mathrm{du}$ collectionneur $\mathrm{S}$. Chtchoukine et des relations entre commanditaires et artistes.

Mais ce travail mené en classe a permis aussi de constater que les élèves étaient intéressés par les œuvres d'art, qu'ils étaient capables de tenir un discours sur celles-ci et qu'ils éprouvaient du plaisir à fabriquer des supports pour les faire partager. Certes, les textes des élèves, dans leur évocation du tableau d'H. Matisse, sont souvent maladroits, hésitants, traduisent les difficultés à dire l'artistique (Chabanne \& Dufays, 2011), mais beaucoup comportent des remarques intéressantes (sans doute insuffisamment exploitées dans la démarche choisie) et traduisent une sensibilité à l'œuvre. De plus, le parti pris de faire écrire les élèves a sans aucun doute augmenté la difficulté de l'exercice pour certains d'entre eux, dans la mesure où il ne fallait pas seulement dire l'artistique, mais 
l'écrire. Mais ce parti-pris relève bien d'un objectif d'ordre esthétique et d'une éducation de la sensibilité.

La longueur de cette séquence - six séances, souvent très longues, et cela sans prendre en compte le temps passé au dépôt des doubles pages sur le site internet de l'école - nous interroge cependant quant à l'ambition des programmes de cycle 3 à propos de l'éducation artistique. Si l'enjeu est d'importance, notamment pour les élèves dont les familles sont éloignées de la culture artistique, comment tenir les objectifs ambitieux mentionnés en histoire des arts? Comment pratiquer l'interdisciplinarité visant le développement de la culture artistique des élèves, autrement que par projets ponctuels et finalement nécessairement rares? Il nous semble nécessaire de faire de cette donnée temporelle l'objet de futures recherches.

\section{BIBLIOGRAPHIE}

ANDREWS, S. et al. (2012). Matisse, serial painter! Paris : Éd. Arola.

Barcilon, M. \& LoRANT-Joly, A. (2013). « Éditions Palette... Rencontre avec Didier Baraud, Céline Delavaux, Christian Demilly ». La revue des livres pour enfants 272, p. 136-142.

BAURET, G. (1977). « La peinture et son commentaire : le métalangage du tableau ». Littérature 27, p. 25-34.

BORDEAUX, M.-C. (2008). « La médiation culturelle en France, conditions d'émergence, enjeux politiques et théoriques ». Colloque international sur la médiation culturelle. Montréal, 4-5 décembre 2008, Culture pour tous/UQAM. En ligne : https://www.lmac-mp.fr/telecharger.php?id_doc=192. CAILLET, E. et al. (dir.) (2016). La Médiation culturelle. Cinquième roue du carrosse ? Paris : L'Harmattan. CHABANNE, J.-C. \& DUfAYS, J.-L. (2011). « Parler et écrire sur les œuvres littéraires et artistiques : contours et enjeux d'une problématique ». Repères 43, p. 7-29. En ligne : http:// journals.openedition.org/pratiques/1150.

CURTIL, S. (2009). « 20 questions pour commencer... ». La revue des livres pour enfants 246, p. 96-97. GALLUZzI, P. (2000). « Les nouvelles technologies et l'éducation hors les murs ». In : Galard, J. (dir.), Le regard instruit. Action éducative et action culturelle dans les musées. Actes du colloque organisé au musée du Louvre, le 16 avril 1999. Paris : La Documentation française, p. 129-142.

HALTÉ, J.-F. (2008). « Le français entre rénovation et reconfiguration ». Pratiques 137-138, p. 23-38. En ligne : http://journals.openedition.org/pratiques/1150.

LARROCHE, C. (2012). Matisse. Paris : Palette.

LECLAIRE-HALTÉ, A. \& MAISONNEUVE, L. (2016). « L'album de littérature de jeunesse : genre, forme et/ ou médium scolaire? » Recherches 65, p. 49-64.

MAtisse, H. (2012) [1908]. Notes d'un peintre. Paris : Éd. du Centre Pompidou.

MINISTÈRE DE L'ÉDUCATION NATIONALE (MEN). (2015). Programmes d'enseignement du cycle des apprentissages fondamentaux (cycle 2), du cycle de consolidation (cycle 3) et du cycle des 
approfondissements (cycle 4). Arrêté du 9-11-2015 - J.O. du 24-11-2015. Paris : MENESR - DGESCO

MAF 1.

MONTANDON, F. \& PEREZ-ROUX, T. (2014). « Introduction ». In : Montandon, F., \& Pérez-Roux, T (dir.), Les médiations culturelles et artistiques. Quels processus d'intégration et de socialisation? Paris :

L'Harmattan.

RIGAT, F. (2005). « Les textes expographiques : pour une approche de la langue-culture dans les expositions d'art moderne ». Études de linguistique appliquée 2, 138, p. 153-170. En ligne : https:// www.cairn.info/revue-ela-2005-2-page-153.htm.

SCHNEIDER, P. (1992) [1984]. Matisse. Paris : Flammarion.

SELLIER, M. (2009). « Pour l'amour de l'art ». La revue des livres pour enfants 246, p. 125-132.

SELLIER, M. (2012). Mon petit Matisse. Paris : Réunion des musées nationaux.

SOUCHIER, E. (1998). « L'image du texte pour une théorie de l'énonciation éditoriale ». Les cahiers de médiologie 6, p. 137-145. En ligne : http://www.cairn.info/revue-les-cahiers-de-

mediologie-1998-2-page-137.html.

TISSERON, S. (1998). « Qu'est-ce qu'une image emblématique ?». Paris : Atelier de l'INA, 4.

VAUGEOIS, D. (2005). « Nom de genre : le nom. De l'usage des “écrits sur l'art" ». In : Vaugeois, D.

(dir.), L'écrit sur l'art. Un genre littéraire? Pau : Publications de l'université de Pau, p. 13-32.

\section{NOTES}

1. Dans le cadre des Lieux d'éducation associés (LéA) à l'IFÉ (ENS de Lyon), les deux auteurs de l'article appartiennent depuis 2016 à un groupe de recherche intitulé « Lire et comprendre des documents composites au cycle $3 »$.

2. Les textes illustrés, par opposition aux iconotextes, caractérisés par une relative contemporanéité de la production du texte et de l'image, se définissent ainsi : « soit le texte, soit l'illustration, sont autonomes selon l'ordre de production de l'un ou de l'autre (texte en premier, illustration en second ou l'inverse)» (Leclaire-Halté \& Maisonneuve, 2016, p. 55). Dans le cas des albums sur les peintres, les œuvres picturales servant d'illustration au texte préexistent à ce dernier, sont interprétables indépendamment du texte même.

3. Les titres de ces deux numéros sont l'Art le livre et les enfants $\left(n^{\circ} 246\right)$ et Création artistique et médiations culturelles en bibliothèque ( $\left.\mathrm{n}^{\circ} 272\right)$.

4. On retrouve cette idée dans les propos mêmes d'H. Matisse $(1908$, p. 27) : « Tout ce qui n'a pas d'utilité dans le tableau est, par la même, nuisible ».

5. Nous remercions I. Aubertin, enseignante à l'école Bourgchevreuil de Cesson-Sévigné (35), d'avoir mis en place dans sa classe la séquence élaborée avec sa collaboration et d'avoir ainsi permis le recueil des données utilisées plus loin.

6. Pour une présentation de cette maison d'édition, lire l'entretien réalisé par M. Barcilon et A. Lorant-Joly (2013) avec le directeur de la maison Palette et deux de ses auteurs dans La revue des livres pour enfants.

7. Les jeux de mots dans les titres sont une caractéristique de cette collection : voir par exemple Keith Haring Révolution'art, Basquiat le grand Bazar, Mr Hopper Mystère Hopper...

8. P. Schneider est l'auteur d'un ouvrage de référence sur H. Matisse.

9. H. Matisse, d'ailleurs, a toujours revendiqué un lien très fort entre la vie d'un artiste et sa production. Il explique ainsi plusieurs fois que ses voyages et ses rencontres ont été pour lui source d'inspiration. 
10. Dans cette classe, deux élèves ont vu, antérieurement à la séquence évoquée, l'exposition parisienne consacrée à la collection S. Chtchoukine qui s'est tenue à la fondation Louis Vuitton en 2016-2017.

11. Nous renvoyons aux nombreux numéros de Pratiques consacrés aux rapports entre lecture et écriture.

12. L'enseignante distingue, dans les textes de ces doubles pages, une dimension descriptive (les textes sont redondants par rapport au tableau), une dimension informative (les textes apportent des informations sur le peintre et son tableau), enfin une dimension interprétative (les textes prennent position par rapport au tableau).

\section{RÉSUMÉS}

L'article s'intéresse, dans un premier temps, aux albums documentaires sur les peintres qui paraissent en littérature de jeunesse, et, dans un second temps, à une séquence interdisciplinaire réalisée dans une classe de $\mathrm{CM} 2$ à partir de ces albums. Il propose d'abord une analyse de trois doubles pages consacrées à La danse, un tableau d'H. Matisse, prises dans trois albums pour la jeunesse différents, et traite de la mise en page de ces trois documents, mais aussi des trois dimensions, informative, interprétative et subjective, des textes, associés à la reproduction du tableau, que l'on peut y lire. Puis il traite de la séquence interdisciplinaire, menée avec un double objectif de la part de l'enseignante : la compréhension du support (objectif lié à la maitrise de la lecture) et la sensibilisation aux œuvres d'art. Après avoir pris connaissance des doubles pages consacrées à ce tableau selon un protocole évoqué dans l'article, des élèves du CM2 observé ont été conviés à fabriquer leur propre double page pour le site internet de l'école. L'article montre comment ils réinvestissent les dimensions des albums dans leur propre écriture et réalisation, dans la mise en page de leurs propositions mais aussi dans leurs textes, alors que ceux-ci n'ont pas fait l'objet d'un travail spécifique.

The article first focuses on the documentary albums on painters published in children's literature and, in a second part, on an interdisciplinary sequence in a CM2 class based on these albums. It first presents an analysis of three double pages devoted to La danse, a painting by H. Matisse, taken from three different youth albums, and deals with the layout of these three documents and the three dimensions of the texts associated with the reproduction of the painting: the informative, interpretive and subjective dimensions, which can be read in those texts. It then deals with the interdisciplinary sequence, carried out with a double objective on the part of the teacher: the understanding of the support (objective linked to proficiency in reading) and the awareness of works of art. After reading the double pages devoted to this picture according to a protocol mentioned in the article, pupils of the observed CM2 class were invited to create their own double page for the Web site of the school. The article shows how they reinvest the dimensions of the albums in their own writing and realization, in the layout of their proposals but also in their texts, even though they have not been the subject of a specific work 
INDEX

Mots-clés : interdisciplinarité, compréhension-lecture documentaire, histoire des artslittérature de jeunesse

Keywords : interdisciplinarity, reading comprehension, documentary history, art history, children's literature

\section{AUTEURS}

\section{ANNE LECLAIRE-HALTÉ}

Université de Lorraine, CREM, EA 3476, ESPÉ de Lorraine, F-57000, France

\section{LUC MAISONNEUVE}

Université de Bretagne occidentale/Université Rennes 2, CREAD, EA 3875, ESPÉ de Bretagne, F-29000, France 